Neural, Parallel, and Scientific Computations 28 (2020) No.4, 273-304

\title{
AN ANALYSIS OF THE RECREATIONAL USE OF MARIJUANA AMONGST THE 21+ POPULATION OF THE STATE OF WASHINGTON IN THE CONTEXT OF I-502 AND ITS AFTERMATH
}

\author{
YUSRA BIBI RUHOMALLY, MUHAMMAD ZAID DAUHOO, AND LAURENT DUMAS \\ Department of Mathematics, Faculty of Science, University Of Mauritius, Reduit, \\ Mauritius. \\ Department of Mathematics, Faculty of Science, University Of Mauritius, Reduit, \\ Mauritius. \\ Laboratoire de Mathématiques de Versailles, UVSQ, CNRS, Université \\ Paris-Saclay, 78035 Versailles, France.
}

\begin{abstract}
This paper analyses the impact of I-502 implementation in the state of Washington, using the NERA model. The NERA system comprises of 4 classes of users namely, N: nonusers, E: experimental users, R: recreational users and A: addicts. The persistence of illicit drug use is proved when the conditions $R_{0}>1$ and $\mu>1$ are ensured. Data available in [33, from 2011 to 2015, is opted for estimating the values of the coefficients for the model. Genetic algorithm is performed and the best coefficients with minimum sum of squared error are obtained. The numerical results indicate a rise in the peer pressure that recreational users and experimental users exert on nonusers to try drugs in the post legalisation period. The concept of targeted campaigns of prevention is explained and a numerical study of the effectiveness of such campaigns of prevention, enacted before I-502, is conducted. The results show a decline in the proportion of experimental, recreational and addict users by $1 \%, 8 \%$ and $3 \%$ respectively. The latter numerical simulation reveals that targeted campaigns of prevention prior to legalisation can help to attenuate abusive use of that drug.
\end{abstract}

AMS (MOS) Subject Classification. 39A10.

\section{Introduction}

With the changing landscape of marijuana laws in the United States, there is an ongoing debate over whether these laws have increased the consumption of marijuana. To date, 11 states and the District of Columbia have legalised recreational use of marijuana and 34 states allow the use of medical marijuana with an appropriate license. In [1], it is reported that more than 55 million American adults have used marijuana in any given month. It is noteworthy that the majority of these users are recreational since there are only about 2 million medical marijuana patients in the United States.

Received August 5, 2020

www. dynamicpublishers . org;

https://doi.org/10.46719/npsc20202844.
1061-5369 \$15.00 (C)Dynamic Publishers, Inc.

www.dynamicpublishers.com; 
It is imperative to differentiate between recreational marijuana legalisation and medical marijuana legalisation. States that have legalised medical marijuana have passed laws that give patients access to marijuana for medical treatment purposes. Most states that allow the use of medical marijuana have a state registration system that issues medical marijuana cards to registered patients. The amount of marijuana that a patient can legally possess varies from state to state [2]. Prior studies have shown that medical marijuana is effective for its therapeutic effects. Patients with diseases namely, AIDS, cancer, chronic pain, persistent muscle spasms, severe nausea, among others use marijuana [2]. In [3], it is stated that medically prescribed marijuana relieves pain in patients with advanced cancer and may also inhibit the growth of tumor cells. Additionally, it has been proven that marijuana use prevent vomiting in cancer chemotherapy patients. It is also remarkable that more than $62 \%$ of people who use medical marijuana is for treating chronic pain [4].

It should be stressed that recreational marijuana consumption differs from medical marijuana use. Recreational use is the consumption intended to alter the user's state of consciousness, with the goal of achieving some sense of euphoria or relaxation [1]. Further, a recreational user seeks to achieve an improved state of mind, whereas the medicinal user seeks to treat a neurotransmitter imbalance that hinders their ability to function normally. The present study deals with the abusive use of marijuana consumption for recreational purposes only.

The recreational use of marijuana among youth is a growing concern to the world [5]. The crucial issue to address is how to deal with recreational users because they are those who then become the advocates of drug consumption and eventually addicts very often. Current prevention strategies are insufficient to address this social epidemic. Therefore, modification of existing drug interventions and the development of new drug policies to better respond to this complex phenomenon is crucial. In [6], it is reported that one cannot draw firm conclusions when relying only on national collected data. Moreover, it is also stated that distinct figures representing the frequency of usage and the amount consumed will eventually provide insights on the evolution of this social epidemic, thereby giving a more accurate picture of the trends.

In November 2012, the state of Washington passed Initiative 502 (I-502) to legalise recreational marijuana use for individuals aged 21 years and older. Since then, six additional states and the district of Columbia have followed suit and all of these states' have restricted legal access to recreational marijuana to those 21 years and older [7]. It should be highlighted that the revenues collected from marijuana taxes, fees and penalties were used to fund public education campaigns, evidence-based prevention and treatment programs [8]. Therefore, several campaigns were created with the fundings obtained from I-502 and as a result, campaigns did not begin until two years after legalisation [6]. This is a paradoxal attempt to curb the propagation of 
illicit drug use because it is typically enhancing the proliferation of a contagious epidemic in a population and then start a campaign of vaccination to prevent it from becoming a pandemic.

Primarily, in 2014, the Department of Health (DOH) of the Washington State has built up a 'Youth Marijuana Prevention campaign' to encourage parents to communicate with their children about the disapproval of using marijuana. In 2016, this prevention and education campaign has directly targeted young people aged $12-$ 17 [9]. Another media-based education campaign, the 'Listen2YourSelfie', targeted youth aged 17-21. The aim of the campaign was to educate youth about the risks of marijuana and empower them to make healthy choices with the slogan: "Remember what's important, Forget marijuana" [10]. Recently, in April 2018, the DOH of the Washington State launched a new campaign, 'You Can', which is the next step of the 'Listen2YourSelfie' campaign, in the aim of disseminating information about the consequences and risks of consuming marijuana. Also, school-based health providers and staff are involved in this campaign. In addition, the latter campaign encourages young people to pursue their future dreams and helps them to see the fatal face of marijuana use that may distract them from their goals [11].

In the report of [12], it is revealed that those who initiate marijuana use at a younger age are four times more likely to become addicted if they use marijuana as a teen. Thus, delaying or preventing the first use of illicit drugs is crucial. These works mentioned above highlight the importance and effectiveness of campaigns of prevention targeted at experimental users, in thwarting illicit drug consumption. Additionally, in [13], it is stated that the United States have relied on the school-based program namely, D.A.R.E, which have been shown to have limited effectiveness. In [14], it is reported that the trends in marijuana use before and after legalisation in Colorado and Washington showed that the proportion of youth reporting past 30-day use has decreased or stayed fairly steady over time. It should be underlined that no significant differences were observed when compared to states without recreational marijuana legalisation. Further, it is argued that prevention should aim to reduce harm and not prevent all use [15]. These observations shed light on the importance of implementing targeted campaigns of prevention which focus on preventing the experimental and recreational users from initiating nonusers into marijuana use.

On 17 October 2018, Canada became the second country to legalise the possession, consumption, and licensed sale of cannabis, after Uruguay. It is not surprising to mention that Canada is already experiencing cannabis shortages [16]. Here, there is evidence that legalisation encourages the experimental use of the drug. Later on, our numerical experiment will confirm a similar situation for the state of Washington. That is, it is shown that there is an increase in the population of experimental users 
just after the enactment of I-502. Moreover, the Government of Canada has committed close to 46 million dollars over the next five years for cannabis public education and awareness activities. These are to inform Canadians, especially youth, of the health and safety risks of cannabis consumption [17. Thus, it is vital for the government and policy makers to be aware of how best to implement awareness campaigns on marijuana use so that the proliferation of recreational and abusive marijuana use, does not increase after the legalisation.

Due to the recent changes in marijuana policies in many states, there are not many studies being done on the impact of marijuana use among adolescents prior to and post the legalisation. Hence, the present study aims to evaluate the effect of targeted campaigns of prevention on both the experimental and the recreational users in the state of Washington, using the NERA model.

\section{Some Existing Dynamic Models of Illicit Drug Consumption}

The new trends in illicit drug consumption is creating new challenges for both researchers and scientists in monitoring and investigating this social epidemic effectively. Extensive research has been going on in modelling the evolution of illicit drug usage and several drug models have been formulated to explore this social dynamic. Despite such advances in research along with the implementation of new drug policies, illicit drug consumption is still skyrocketing around the globe and is causing drastic consequences to the youth. The challenge comes largely from the fact that it is difficult to acquire data on illicit drug usage from the individuals as they are unwilling to give out such information [18]. Consequently, it poses many difficulties to both scientists and the government to address and control this complex problem effectively. Extensive, qualitative and quantitative research into this phenomenon is essential.

As mentioned in [18, dynamic drug models are an interesting approach to gauge the behaviour of this phenomenon under different circumstances, by varying distinct parameters of the system and investigating the corresponding outcomes. This semiexperimental process of modifying certain parameters of the model and evaluating the resulting outcomes is called 'what if' scenario analysis [19]. It is noteworthy that policy makers and scientists are seeking answers to several questions concerning the emerging rise of illicit drug use. For instance, the questions are as follows: "what trends are emerging following a legalisation in a certain population?', 'which policies are more effective?' and 'what are the associated costs related to the implemented policies?' [20]. Hence, mathematical modelling can be an efficient predictive tool and as such becoming a useful device to aid researchers in designing and choosing appropriate intervention drug measures. Also, through dynamic models, researchers can simulate policy experiments that are not possible in real life due to practical reasons 
since performing experimentation on a human population is often impossible, unethical and expensive [21]. The work of [22], 23], [18] and [24] are notable examples where the authors have analysed this social epidemic using the NERA model.

The main objective of the present work is to use the NERA model in order to assess the impact of I-502 on recreational marijuana consumption, in the state of Washington. It should be stressed that this work does not address the question of whether legalisation is the solution or not, it instead focuses on the design of how best to implement legalisation in a certain population by implementing targeted campaigns of prevention to the youth.

The paper is organised as follows: Section 3 describes the NERA system while Section 3.2 presents an analysis of the model. The permanence of illicit drug use is proved in Section 3.3. Section 4 culminates into the verification of the NERA model. Data available in 33 ] is opted for the verification of the model and genetic algorithm is conducted for the periods pre and post I-502, in the state of Washington. The effect and a quantitative analysis of the enactment of targeted campaigns of prevention before and after I-502, is examined thoroughly in Sections 5 and 6. Certain limitations pertaining to the present study are given in Section 7 and the concluding remarks are eventually provided in Section 8 .

\section{The NERA Model}

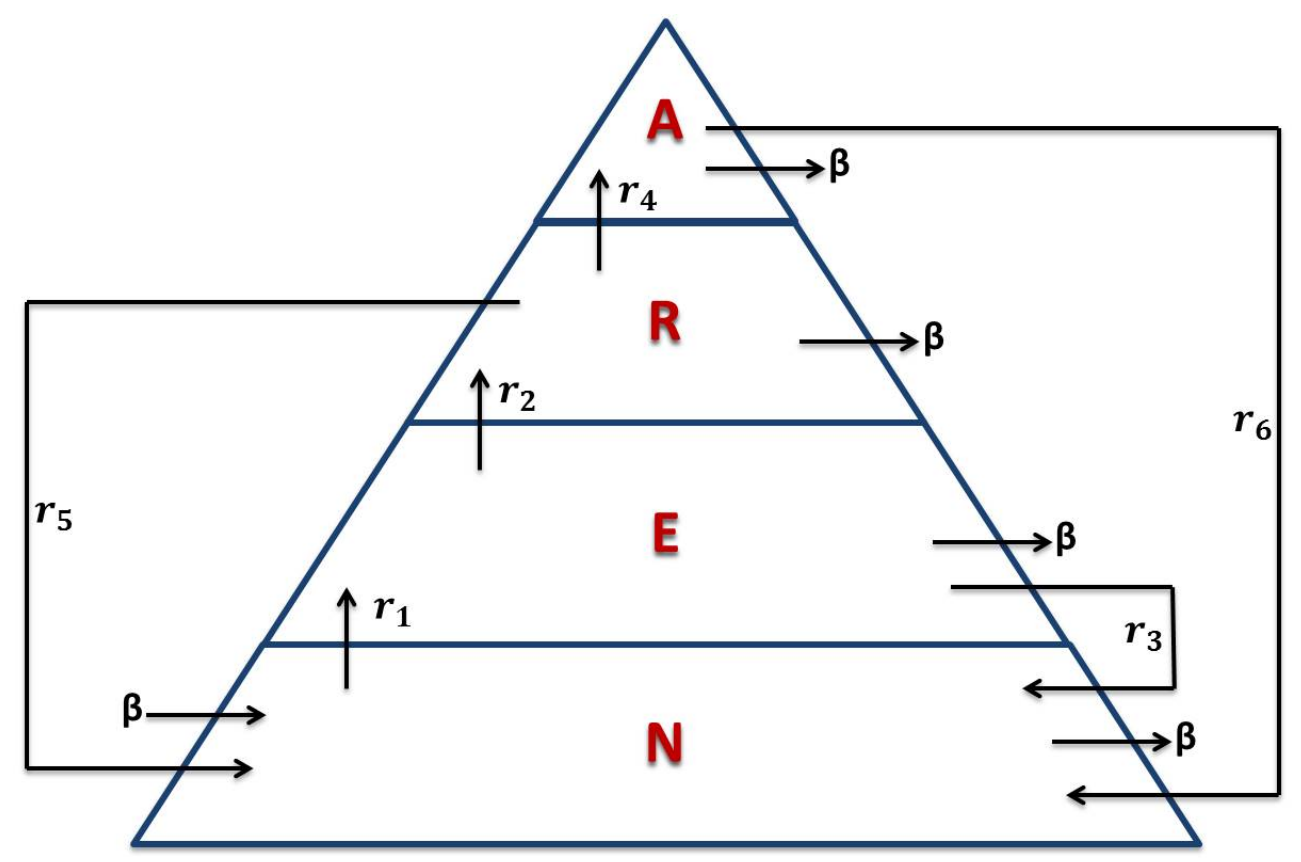

FiguRE 1. Schematic representation of the NERA model.

The NERA system is a four compartmental model, consisting of both drug users and nonusers $(\mathrm{N})$. Three classes of illicit drug users are considered, namely, experimental 
users $(E)$, recreational users $(\mathrm{R})$ and addicts $(\mathrm{A})$. The proportion of each category at a particular time, $t$ is represented as $N(t), E(t), R(t)$ and $A(t)$ respectively, such that

$$
N(t)+E(t)+R(t)+A(t)=1,
$$

where the total population is normalised to 1 unit. Figure 1 depicts a schematic representation of the model. The transition of an individual from a class to another is represented by the different arrows, as shown in Figure 1. More details of the NERA model can be found in [22, [23] and [18].

The following system of ordinary differential equations delineates the dynamics of $N(t), E(t), R(t)$ and $A(t)$.

$$
\begin{aligned}
\frac{\mathrm{d} N(t)}{\mathrm{d} t} & =r_{5} R(t)+r_{3} E(t)+r_{6} A(t)+\beta-\beta N(t)-r_{1} N(t) R(t)-r_{1} N(t) E(t), \\
\frac{\mathrm{d} E(t)}{\mathrm{d} t} & =r_{1}(E(t)+R(t)) N(t)-r_{2} R(t) E(t)-\left(\beta+r_{3}\right) E(t), \\
(3.1) \frac{\mathrm{d} R(t)}{\mathrm{d} t} & =r_{2} R(t) E(t)-\left(\beta+r_{4}+r_{5}\right) R(t), \\
\frac{\mathrm{d} A(t)}{\mathrm{d} t} & =r_{4} R(t)-\left(\beta+r_{6}\right) A(t) .
\end{aligned}
$$

Table 1 gives the definition of all the parameters involved in the system.

TABLE 1. Interpretation of the parameters in the NERA model.

\begin{tabular}{ll}
\hline \hline Parameter & Physical Meaning \\
\hline$r_{1}$ & Influence rate of $E(t)$ and $R(t)$ on $N(t)$ \\
$r_{2}$ & Influence rate of $R(t)$ on $E(t)$ \\
$r_{3}$ & Rate at which experimental users quit drugs \\
$r_{4}$ & Rate at which recreational users change to addicts \\
$r_{5}$ & Rate at which recreational users quit drugs \\
$r_{6}$ & Rate at which addicts quit drugs \\
$\beta$ & Rate of moving in or out of the population due to ageing \\
\hline
\end{tabular}

3.1. The versatility of the NERA model. Mathematical modelling of infectious diseases unearth the complexities underlying the infection and furnish invaluable insights into the evolving mechanisms that drive disease invasion and spread. To obtain a swift and a first approximation of the proliferation of a disease, it is adequate to use a version of the classical SIR model [25]. Nonetheless, with major outbreaks emerging in the world namely, swine influenza, SARS, Ebola and more recently the Covid-19 pandemic, have fuelled the need for a more precise description of the disease dynamics that emanates predictive power to be used for implementing measures for disease extinction, suppression of its spread and control [26]. 
A major assumption often employed in mathematical models that mimic disease dynamics is that the recovery state is attained only after the final state of infection. It is important to note that the progression of an infectious disease is stratified into five periods namely, the incubation, prodromal, illness, decline, and convalescence periods. These periods are categorised by the severity of signs and symptoms [27]. Accordingly, since the NERA system is partitioned into subcategories that depend on the frequency of drug use and which is alike to the severity of signs and symptoms of an infectious disease, the latter system can be easily adapted to model numerous infectious diseases.

It should be accentuated that unlike the basic compartmental models where the users join the recovered class only after the final state of infection, in the NERA system the drug users can join the nonuser class after being in any of the infected states. In addition, it should be stressed that in the modelling of infectious diseases, numerous studies violate the fact that infected individuals with mild symptoms can also recover. Such practical and reasonable fact is often seen to be neglected when setting up a mathematical model as a single class of infected individuals is commonly incorporated in the established model. For instance, in the context of the Covid-19 pandemic, it is underscored that individuals with mild symptoms can also worsen the spread of the disease, making it challenging to contain the spread. Moreover, infected individuals of the Covid-19 pandemic have been categorised as mild, severe and critical [28]. Further, it should be highlighted that these 3 cases of infected individuals have seen to recover [28] [29].

In light of the above, these 3 categories are seen to reflect the dynamics of the three infected classes that are embodied in the NERA system. Therefore, the NERA system is perceived to be highly flexible to model any infectious disease. With the NERA framework, various targeted strategies can be devised and enacted in order to better understand the continuously changing dynamics of an outbreak. Consequently, these features make the NERA model different from the basic compartmental models as the system can capture the key features that are responsible for the spread of any contagious disease in a certain population. In a certain sense, the versatility of the NERA model is paving the way to a more detailed study of epidemics.

The next subsections deal with the analysis of the NERA system and it is shown that the model exhibits uniform permanence of illicit drug use, under certain conditions.

3.2. Analysis of the NERA model. This NERA framework has been first analysed and investigated in [22] to explore the dynamical behaviour of the evolution of illicit drug use within a given population. The authors analysed the nonlinear dynamical system as illustrated by system (3.1), using data available in 30]. 
The latter work was revisited in [23], where the nature of the solution of the NERA model was studied. The uniqueness, positivity and boundedness of the solution was proved. The main focus was the formulation of a stochastic system, called SNERA. The model was extended by taking into account the unpredictability of person-toperson contact. That is, the stochastic system takes into account the social interactions that are responsible for the drug use spread within drug users' community which caters for the stochastic aspect of drug experimentation.

Recently, the NERA model has been further scrutinised in [18. System (3.1) is analysed in the region

$$
\Omega=\left\{(N, E, R, A) \in \mathbb{R}_{+}^{4}: 0 \leq N+E+R+A \leq 1\right\}
$$

which is a positively invariant set.

The model has the following 3 equilibria:

1. The Drug Free Equilibrium, (DFE), $P_{0}=(1,0,0,0)$ which represents a population free of drug users.

2. The Recreational Addict Free (RAF) equilibrium, $P^{*}=\left(N^{*}, E^{*}, R^{*}, A^{*}\right)$ where,

$$
N^{*}=\frac{\beta+r_{3}}{r_{1}}, \quad E^{*}=\frac{r_{1}-r_{3}-\beta}{r_{1}} \quad \text { and } \quad R^{*}=A^{*}=0 .
$$

As mentioned in [22], $P^{*}$ is known as the RAF equilibrium since the population consists only of nonusers and experimental users.

3. The Drug Endemic Equilibrium (DEE), $P^{* *}=\left(N^{* *}, E^{* *}, R^{* *}, A^{* *}\right)$ which consists of all the four categories of drug users.

In order to analyse the model qualitatively, two threshold terms namely, $R_{0}$ and $\mu$ of model (3.1) are derived that deduce when consumption of drugs is more probable to die out or persist in a population. The first reproduction number, $R_{0}$ for the system is given as

$$
R_{0}=\frac{r_{1}}{\beta+r_{3}}
$$

which is defined as the average number of individuals from the nonuser category, who are influenced by a member of the drug user population to become experimental users and recreational users.

In addition, the analysis conducted in [18] revealed that when $R_{0}<1$, the DFE is both locally and globally assymptotically stable. However, as $R_{0}>1$, the DFE loses its stability and becomes unstable. $R_{0}>1$ gives rise to the appearance of two possible endemic equilibria namely, the RAF equilibrium and the DEE. The two endemic equilibria are stable when $R_{0}>1$, under certain conditions. 
Since $R_{0}=\frac{r_{1}}{\beta+r_{3}}$,

$$
R_{0}>1 \Longrightarrow r_{1}>\beta+r_{3}
$$

An epidemiological interpretation of $R_{0}>1$ is that the average number of new drug users in the experimental category due to the influence of an experimental user or a recreational user on the nonuser category is more than one. In other words, each individual of the experimental and recreational categories is influencing more than one nonuser to initiate drug use in order to join the experimental category. This can be a feature of societies in which marijuana is legally recreational unless proper campaigns of prevention are implemented. The DFE will no longer exist when $R_{0}>1$ since the experimental and the recreational users exert a maximum influence on the nonusers to try drugs. As a result, another endemic equilibrium emerges and persists in the population.

The second reproduction number, $\mu$ is given as

$$
\mu=\frac{r_{2}\left(r_{1}-r_{3}-\beta\right)}{r_{1}\left(\beta+r_{4}+r_{5}\right)} .
$$

In terms of $R_{0}, \mu$ can be written as

$$
\mu=\frac{r_{2}\left(r_{1}-r_{3}-\beta\right)}{r_{1}\left(\beta+r_{4}+r_{5}\right)}=\left(\frac{R_{0}-1}{R_{0}}\right) \frac{r_{2}}{\left(\beta+r_{4}+r_{5}\right)} .
$$

From Table 1, $r_{2}$ represents the influence rate that recreational users exert on the experimental category. A recreational user is expected to spend on average, $\frac{1}{\left(\beta+r_{4}+r_{5}\right)}$ time units in the recreational category. Heuristically, $\mu$ can therefore be defined as the influence that a recreational user exert on the experimental users during its lifetime in the recreational category.

When $R_{0}>1$ and $\mu<1$, the RAF equilibrium is locally assymptotically stable while the DEE is unstable. That is, the population is recreational addict free.

Moreover, it should be remarked that

$$
\mu=\left(\frac{R_{0}-1}{R_{0}}\right) \frac{r_{2}}{\left(\beta+r_{4}+r_{5}\right)}<\frac{r_{2}}{\left(\beta+r_{4}+r_{5}\right)} .
$$

Therefore,

$$
\mu>1 \Longrightarrow \frac{r_{2}}{\left(\beta+r_{4}+r_{5}\right)}>1 \Longrightarrow r_{2}>\left(\beta+r_{4}+r_{5}\right)
$$

Epidemiologically speaking, from inequality (3.9), $\mu>1$ means that the influence rate of recreational users on the experimental users increases to become larger than 1. In other words, each recreational user is influencing more than one experimental user during its lifetime in the recreational category. As a result, this renders the RAF 
equilibrium to be unstable and indicates the appearance of the DEE, where all the four categories coexist in the population.

Additionally, when $r_{2}>r_{1}, \mu$ increases significantly. That is, the recreational users exert a stronger influence on the experimental category as compared to the influence that they exert on the nonusers. Such a situation where drug users may influence experimental users significantly can occur post the legalisation of certain illicit drugs in a certain population. Thus, when $R_{0}>1$ and $\mu>1$, both the DFE and the RAF equilibrium are unstable while the DEE is stable.

From the definition of $R_{0}, \mu$ and the latter inferences, it can be concluded that in order to eradicate illicit drug consumption in a given population, the basic reproduction number, $R_{0}<1$. This threshold term becomes less than 1 when the parameter $r_{1}$ is reduced and the parameter $r_{3}$ is increased accordingly.

Next, the permanence of illicit drug use in the NERA model when $\mu>1$ and $R_{0}>1$, is shown.

3.3. Persistence of drug consumption. The study of uniform persistence of model (3.1) requires the introduction of the following sets:

$$
\begin{aligned}
\Omega & =\left\{(N(t), E(t), R(t), A(t)) \in \mathbb{R}_{+}^{4}: N(t)+E(t)+R(t)+A(t) \leq 1\right\}, \\
(3.10) \Omega_{0} & =\{(N(t), E(t), R(t), A(t)) \in \Omega: E>0, R>0, A>0\}, \\
\partial \Omega_{0} & =\Omega \backslash \Omega_{0},
\end{aligned}
$$

We next introduce the following Lemma.

Lemma 3.1. Each solution $(N(t), E(t), R(t), A(t))$ of Equations 3.1, with initial conditions $\in \Omega_{0}$ remains in $\Omega_{0}$ for all $t>0$. 
Proof. Solving the system of Equations 3.1 with initial value $(N(0), E(0), R(0), A(0))$, the solutions for $N(t), E(t), R(t)$ and $A(t)$ are given as follows:

$$
\begin{aligned}
N(t)= & e^{-\int_{0}^{t} \beta+r_{1} E(s)+r_{1} R(s) d s} \\
& \times\left[N(0)+\int_{0}^{t}\left(\beta+r_{3} E(s)+r_{5} R(s)+r_{6} A(s)\right) \times e^{\int_{0}^{s} \beta+r_{1} E(c)+r_{1} R(c) d c} d s\right] \\
& >0, \quad \forall t>0, \\
E(t)= & e^{-\int_{0}^{t} \beta+r_{3}+r_{2} R(s)-r_{1} N(s) d s} \\
& \times\left[E(0)+\int_{0}^{t} r_{1} N(s) R(s) \times e^{\int_{0}^{s} \beta+r_{3}+r_{2} R(c)-r_{1} N(c) d c} d s\right]>0, \quad \forall t>0, \\
R(t)= & R(0) \times e^{\int_{0}^{t} r_{2} E(s)-\left(\beta+r_{4}+r_{5}\right) d s}>0, \forall t>0, \\
A(t)= & e^{-\left(\beta+r_{6}\right) t}\left[A(0)+\int_{0}^{t} r_{4} R(s) e^{\left(\beta+r_{6}\right) s} d s\right]>0, \forall t>0,
\end{aligned}
$$

for any $(N(0), E(0), R(0), A(0)) \in \Omega_{0}$, showing that $\Omega_{0}$ is positively invariant.

In view of the above, it is deduced that every trajectory in $\Omega_{0}$ eventually stays in $\Omega_{0} \forall t>0$.

Theorem 3.2. If $R_{0}>1$ and $\mu>1$, then model (3.1) is uniformly persistent, that is $\exists \delta>0$ such that

$$
\lim _{t \rightarrow+\infty} \inf N(t)>\delta, \quad \lim _{t \rightarrow+\infty} \inf E(t)>\delta, \quad \lim _{t \rightarrow+\infty} \inf R(t)>\delta, \quad \lim _{t \rightarrow+\infty} \inf A(t)>\delta
$$

where $\delta>0$ is independent of the initial data in $\Omega$.

In other words, when $R_{0}>1$ and $\mu>1$, if the population consists of all the 4 categories, the latter remain non zero as $t \rightarrow \infty$.

Proof. The persistence theory of dynamical systems in [31] (Section 1.3 in Chapter 1 ) is employed to show that the 4 categories of drug users coexist when $R_{0}>1$ and $\mu>1$.

It is required to prove that system (3.1) is uniformly persistent with respect to $\left(\Omega, \Omega_{0}\right)$. From Section 3 and from Lemma 3.1 , it is seen that both $\Omega$ and $\Omega_{0}$ are positively invariant.

Let $(N(t), E(t), R(t), A(t))$ be the solution of system 3.1 with initial condition $(N(0), E(0), R(0), A(0))$ and we define

$(3 \mathrm{X} 1 Z)\left\{(N(0), E(0), R(0), A(0)) \in \partial \Omega_{0}:(N(t), E(t), R(t), A(t)) \in \partial \Omega_{0}, \forall t>0\right\}$.

Since the DFE is globally asymptotically stable, starting with the initial condition $(N(0), E(0), R(0), A(0))=\left(N_{0}, 0,0,0\right)$, where $N_{0}>0$, then at any time $t$ the system remains in the DFE. 
Similarly, starting with a recreational addict free population and since the RAF equilibrium is locally asymptotically stable and for $t$ sufficiently small, the system stays in the RAF state.

In other words, starting with the initial condition $(N(0), E(0), R(0), A(0))=\left(N_{0}, E_{0}, 0,0\right)$, where $N_{0}>0$ and $E_{0}>0$ and for $t$ sufficiently small, the population remains recreational addict free.

Clearly, the DFE and the RAF equilibrium are found in $X$. That is,

$$
D F E_{1}=\left\{\left(N_{0}(t), 0,0,0\right): N_{0}(t)>0\right\} \subset X
$$

and

$$
R A F_{1}=\left\{\left(N_{0}(t), E_{0}(t), 0,0\right): N_{0}(t)>0, E_{0}(t)>0\right\} \subset X .
$$

It is pointed out that $D F E_{1}$ and $R A F_{1}$ are 2 disjoint sets that is, $D F E_{1} \cap R A F_{1}=\phi$. It is next demonstrated that any element of $X$ is either an element of $D F E_{1}$ or an element of $R A F_{1}$. Thus,

$$
X=D F E_{1} U R A F_{1} .
$$

If $P=(N(t), E(t), R(t), A(t)) \in X$, then at least 1 category of the population does not exist. Therefore, there exists $t_{0}>0$ such that either

(1) $\left(E\left(t_{0}\right)=0, R\left(t_{0}\right)>0, A\left(t_{0}\right)>0\right)$ or

(2) $\left(E\left(t_{0}\right)>0, R\left(t_{0}\right)=0, A\left(t_{0}\right)>0\right)$ or

(5) $\left(E\left(t_{0}\right)=0, R\left(t_{0}\right)>0, A\left(t_{0}\right)=0\right) \in X$.

It is noted that

$$
P \in X \Longrightarrow P \in D F E_{1} \text { or } P \in R A F_{1} \text {. }
$$

Considering the third case for $\left(E\left(t_{0}\right)>0, R\left(t_{0}\right)>0, A\left(t_{0}\right)=0\right)$ and from the fourth equation of model (3.1),

$$
\frac{d A}{d t}=r_{4} R\left(t_{0}\right)>0 \text {. }
$$

It then follows that $\exists \epsilon_{0}>0$ such that $A(t)>0$ for $t_{0}<t<t_{0}+\epsilon_{0}$. By the continuity of $E(t)$ and $R(t)$, we can restrict $\epsilon_{0}>0$ small enough such that $E(t)>0$ and $R(t)>0$ for $t_{0}<t<t_{0}+\epsilon_{0}$.

This explains that $(N(t), E(t), R(t), A(t)) \notin \partial \Omega_{0}$ for $t_{0}<t<t_{0}+\epsilon_{0}$, is contradictory to the assumption that $(N(0), E(0), R(0), A(0)) \in X$.

It is easy to verify that cases (1), (2), (4) and (5) also contradict the assumption that $(N(0), E(0), R(0), A(0)) \in X$. Thus, Equations (3.13) and (3.14) hold. 
It is sufficient to show that $W\left\{P_{0}, P^{*}\right\} \cap \Omega_{0}=\emptyset$ when $R_{0}>1$ and $\mu>1$, where

$$
W\left\{P_{0}, P^{*}\right\}=\left\{\begin{array}{l}
(N(0), E(0), R(0), A(0)): \lim _{t \rightarrow+\infty}(N(t), E(t), R(t), A(t))=P_{0} \text { or } \\
(N(0), E(0), R(0), A(0)): \lim _{t \rightarrow+\infty}(N(t), E(t), R(t), A(t))=P^{*},
\end{array}\right.
$$

is a stable set of $\left\{P_{0}, P^{*}\right\}$.

It is proved that $W\left\{P_{0}, P^{*}\right\} \cap \Omega_{0} \neq \emptyset$ when $R_{0}>1$ and $\mu>1$.

Suppose that there is a point $(N(0), E(0), R(0), A(0)) \in \Omega$ such that

$\lim _{t \rightarrow+\infty}(N(t), E(t), R(t), A(t))=P_{0}$.

Then, for any given $\lambda \in(0,1)$, there exists $t_{0}>0$ such that

$$
\left|N(t)-N_{0}\right|<\lambda, \quad E(t)<\lambda, \quad R(t)<\lambda \text { and } A(t)<\lambda \forall t>t_{0} .
$$

That is, $N_{0}-\lambda<N(t)<N_{0}+\lambda, 0<E(t)<\lambda, 0<R(t)<\lambda$ and $0<A(t)<\lambda$.

The second Equation of model 3.1 becomes

$$
\frac{d E}{d t}>r_{1} E\left(N_{0}-\lambda\right)-r_{2} E \lambda-\left(\beta+r_{3}\right) E .
$$

Since $R_{0}=\frac{r_{1}}{\beta+r_{3}} \Longrightarrow\left(\beta+r_{3}\right)=\frac{r_{1}}{R_{0}}$, Equation 3.17 can be written as

$$
\begin{gathered}
\frac{d E}{d t}>r_{1} E\left(N_{0}-\lambda\right)-r_{2} E \lambda-\left(\frac{r_{1}}{R_{0}}\right) E \\
=\left[r_{1}\left(N_{0}-\lambda\right)-\frac{r_{1}}{R_{0}}-r_{2} \lambda\right] E .
\end{gathered}
$$

Let

$$
g_{1}(\lambda)=r_{1}\left(N_{0}-\lambda\right)-\frac{r_{1}}{R_{0}}-r_{2} \lambda
$$

$g_{1}(\lambda)>0$ provided that

$$
\lambda<\frac{r_{1}}{r_{1}+r_{2}}\left(N_{0}-\frac{1}{R_{0}}\right) .
$$

Since the $D F E=\left(N_{0}, 0,0,0\right)=(1,0,0,0)$,

$$
\lambda<\frac{r_{1}}{r_{1}+r_{2}}\left(1-\frac{1}{R_{0}}\right)<1 .
$$

Therefore, for $0<\lambda<\frac{r_{1}}{r_{1}+r_{2}}\left(1-\frac{1}{R_{0}}\right)<1, E(t) \rightarrow \infty$ as $t \rightarrow \infty$.

That is, $\lim _{t \rightarrow+\infty} E(t)=\infty$, which leads to the contradiction that $\lim _{t \rightarrow+\infty} E(t)=0$.

In other words, there exists a $\lambda \in\left(0, \frac{r_{1}}{r_{1}+r_{2}}\left(1-\frac{1}{R_{0}}\right)\right)$ such that

$$
\lim _{t \rightarrow+\infty}(N(t), E(t), R(t), A(t)) \neq P_{0} \text {. }
$$


It is next supposed that there is a point $(N(0), E(0), R(0), A(0)) \in \Omega$ such that $\lim _{t \rightarrow+\infty}(N(t), E(t), R(t), A(t))=P^{*}$.

That is, starting with a population where all the 4 categories are non zero, then, the population becomes recreational addict free as $t \rightarrow \infty$. That is, $R(t) \rightarrow 0$ and $A(t) \rightarrow 0$ as $t \rightarrow \infty$.

Then, there exists $\gamma \in(0,1)$ such that

$(3.22) N(t)-N_{0}|<\gamma, \quad| E(t)-E_{0} \mid<\gamma, R(t)<\gamma$, and $A(t)<\gamma \forall t>t_{0}$.

That is, $N_{0}-\gamma<N(t)<N_{0}+\gamma, E_{0}-\gamma<E(t)<E_{0}+\gamma, 0<R(t)<\gamma, 0<A(t)<\gamma$ and the third Equation of model 3.1 becomes

$$
\frac{\mathrm{d} R(t)}{\mathrm{d} t}>r_{2}\left(E_{0}-\gamma\right) R-\left(\beta+r_{4}+r_{5}\right) R
$$

Since $\mu=\frac{\left(R_{0}-1\right) r_{2}}{R_{0}\left(\beta+r_{4}+r_{5}\right)} \Longrightarrow\left(\beta+r_{4}+r_{5}\right)=\frac{r_{2}\left(R_{0}-1\right)}{\mu R_{0}}$, Equation 3.23 becomes

$$
\frac{\mathrm{d} R(t)}{\mathrm{d} t}>\left[r_{2}\left(E_{0}-\gamma\right)-\left(\frac{R_{0}-1}{R_{0}}\right)\left(\frac{r_{2}}{\mu}\right)\right] R
$$

Let

$$
g_{2}(\gamma)=r_{2}\left(E_{0}-\gamma\right)-\left(\frac{R_{0}-1}{R_{0}}\right)\left(\frac{r_{2}}{\mu}\right)
$$

$g_{2}(\gamma)>0$ provided that

$$
\gamma<E_{0}-\left(\frac{R_{0}-1}{R_{0}}\right)\left(\frac{1}{\mu}\right)
$$

From [18, the $R A F=\left(N_{0}, E_{0}, 0,0\right)=\left(\frac{1}{R_{0}}, 1-\frac{1}{R_{0}}, 0,0\right)$ and therefore,

$$
\gamma<\left(\frac{R_{0}-1}{R_{0}}\right)\left(1-\frac{1}{\mu}\right)<1 \text { when } \mu>1 \text { and } R_{0}>1 \text {. }
$$

Thus, for $0<\gamma<\left(\frac{R_{0}-1}{R_{0}}\right)\left(1-\frac{1}{\mu}\right)<1, R(t) \rightarrow \infty$ as $t \rightarrow \infty$.

That is, $\lim _{t \rightarrow+\infty} R(t)=\infty$, which leads to the contradiction that $\lim _{t \rightarrow+\infty} R(t)=0$.

In other words, there exists a $\gamma \in\left(0,\left(\frac{R_{0}-1}{R_{0}}\right)\left(1-\frac{1}{\mu}\right)\right)$ such that

$$
\lim _{t \rightarrow+\infty}(N(t), E(t), R(t), A(t)) \neq P^{*} \text {. }
$$

Consequently, this proves that $W\left\{P_{0}, P^{*}\right\} \cap \Omega_{0}=\emptyset$ when $R_{0}>1$ and $\mu>1$.

Hence, by the theorems of uniform persistence for dynamical systems given in [32], it is concluded that model $(3.1)$ is uniformly persistent with respect to $\left(\Omega, \Omega_{0}\right)$. In an epidemiological view, uniform persistence of model 3.1 implies that there are always $E, R$ and $A$ individuals when $R_{0}>1$ and $\mu>1$. 
From these studies, it can be accentuated that the NERA model described above has the capacity to incorporate data about prevention efforts and show its impact on the different transitions to or from illicit drug use over time. Additionally, since the parameter values of the system can be adjusted and corresponding outcomes obtained, the model justifies its use as a policy control mechanism in controlling and tackling illicit drug consumption. It may assist policy and decision makers in setting potential targets of the type of prevention efforts that will be required to impact the transition rates at a level that would help to maintain and mitigate marijuana use in any given population.

It should be pointed out that the primary concern in designing a mathematical model should be its ability to provide adequate results. This implies that verification of a dynamical system is one of the most important factors which has to be considered in the mathematical modelling aspect. Thus, in the present work, the results of the NERA model is verified using data available in [33], on the prevalence of marijuana use in the population of $21+$ in the state of Washington. The evolution of the four categories of drug users is simulated within the state of Washington from 2011 and beyond the implementation of I-502. In addition, since there exists different views over the impact of the decriminalisation, one crucial issue to address is the influence of decriminalisation on experimental and recreational use of drugs.

Hence, the objective of this study is to show the versatility of the model and investigate the impact of I-502 in the state of Washington, quantitatively and qualitatively. Also, the effect of $\mathrm{I}-502$ on the transition rates of the drug users is examined using the system.

The next section culminates into the verification of the NERA model and an investigation on the effect of I-502 in the state of Washington, is conducted. Data available in [33] is opted for the verification of the model, using genetic algorithm for the periods pre and post $\mathrm{I}-502$, in the state of Washington.

\section{Verification of the solution of the NERA model}

The Washington State Institute for Public Policy conducted a benefit-cost evaluation of the implementation of I-502 to analyse and identify the effects on youth and adult substance use, cannabis abuse treatment admissions, and drug-related criminal convictions. It is important to note that the term marijuana is interchangeably referred to as cannabis in the latter report.

The data set available in the report for marijuana use represents the following variables: Lifetime marijuana use, 30-day marijuana use and 30-day heavy marijuana 
use. Figure 2 shows the trends in marijuana use for the three different categories of marijuana users in the $21+$ population, in the state of Washington according to the data gathered in [33]. Within the background of the NERA model, the three variables for marijuana correspond to the experimental, recreational and addict category, respectively.

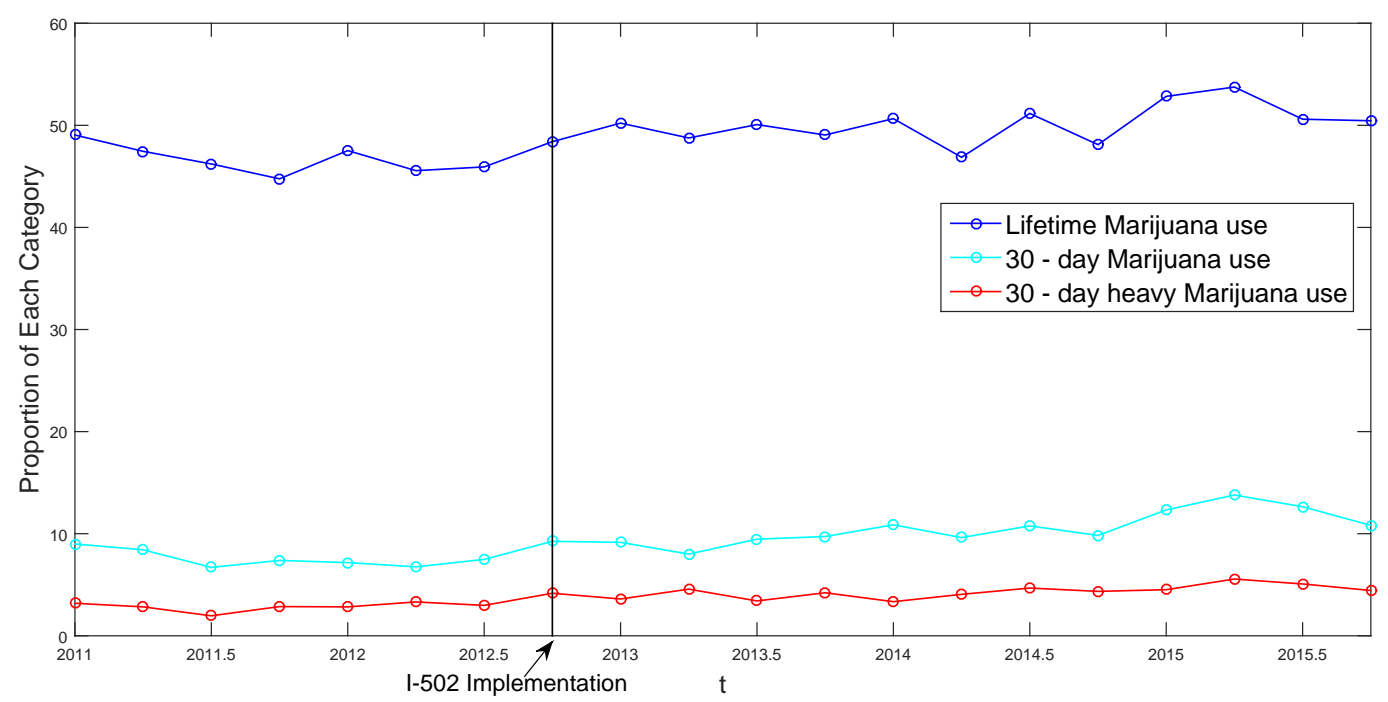

FigurE 2. Trends in Marijuana use in the state of Washington [33].

In the case of the NERA model, the corresponding values for the nonuser category, $N$ are evaluated as follows:

$$
N(t)=1-(E(t)+R(t)+A(t)) .
$$

Figure 3 illustrates the mean proportion of the 4 categories of users, prior to and post the legalisation.

From Figure 3, a general increase in the mean proportion of each category after the implementation of $\mathrm{I}-502$ is observed. This can be explained as just after the
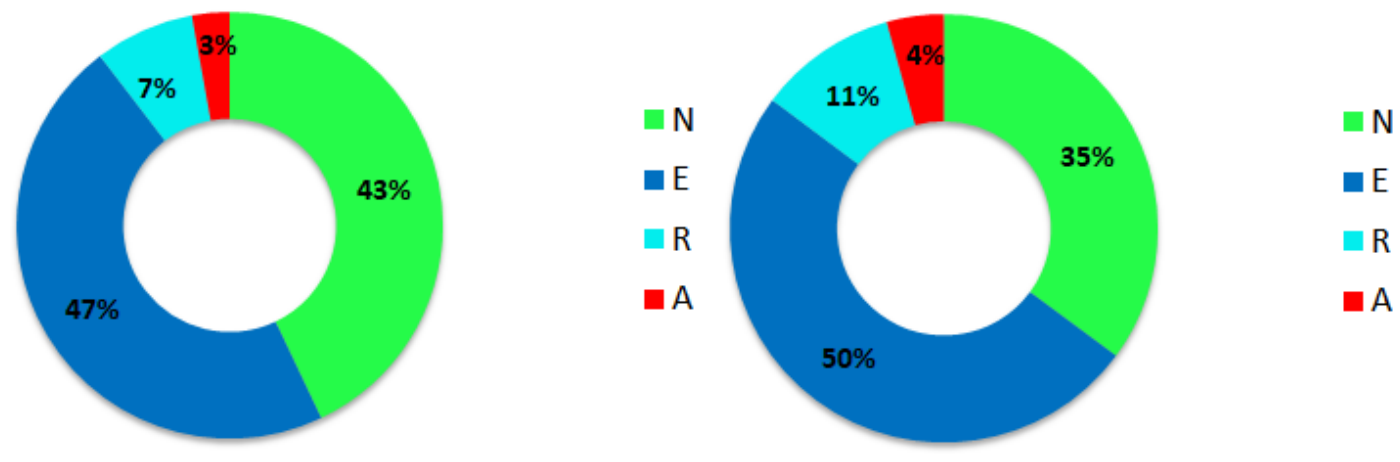

Figure 3. Mean proportion of the 4 categories of users before and after I-502 implementation according to the data gathered in [33]. 
legalisation, the nonusers will experiment the drug to cure their curiosity about the sensations they produce [22]. Also, a rise in the experimental category which is at the expense of a drop of $8 \%$ in the population of nonusers is noted.

In Washington, funding for the respective education campaigns came from the state marijuana revenues. As a result, campaigns of prevention did not begin until two years after legalisation [6]. Thus, this evidence the rise of the number of drug users after the legalisation in November 2012.

In order to use and verify the NERA model, it is required to evaluate the set of parameters given in Table 1. These are computed using the data available in [33]. Genetic algorithm is used to obtain the optimum set of parameters that fits the system. In the next section, the concept of genetic algorithm and the objective function used for the system are explained.

4.1. Estimation of Parameters using Genetic Algorithm. In the case of the NERA model, the fitness function, $f$, is the sum of the squared differences between the collected data and the model prediction.

$$
f\left(r_{1}, r_{2}, \cdots, r_{6}\right)=\sum_{i=1}^{4}\left(D_{i}-P_{i}\right)^{2}
$$

where

$i=1,2,3,4$ correspond to the state variables: $N, E, R$ and $A$ respectively,

$D_{i}$ : the temporal derivative of the best fit line passing through the set of data for each category $i$ over the corresponding period: pre or post I-502

and

$P_{i}$ : the evaluated right hand side function of system 1 using the mean value for each category $i$ over the corresponding period: pre or post I-502

such that the following linear constraints and bounds are satisfied:

$$
\begin{gathered}
\Omega=\left\{(N, E, R, A) \in \mathbb{R}_{+}^{4}: 0 \leq N+E+R+A \leq 1\right\} \\
\epsilon \leq \sum_{j=1}^{6} r_{j} \leq 1, \quad \text { where } \epsilon>0
\end{gathered}
$$


and

$$
0<r_{j}<1, \forall j=1,2, \cdots, 6 \text {. }
$$

For the estimation of the model parameters, the parameter, $\beta$ which represents the rate of moving in or out of the population due to ageing, is kept as $\frac{1}{15}[34]$.

Firstly, the best fit line passing through the set of data points for each category for the period before I-502, is sought. The gradient of each such line, corresponding to an approximation to the temporal variation of each category for the period Jan 2011 to Sept 2012 is then obtained. Table 2 shows the resulted outcomes.

\begin{tabular}{ccccc}
\hline \hline Variable & $\mathrm{N}$ & $\mathrm{E}$ & $\mathrm{R}$ & $\mathrm{A}$ \\
\hline$D_{i}$ & 2.577 & -1.694 & -1.057 & 0.174 \\
\hline
\end{tabular}

TABle 2. Temporal derivatives for the state variables $N, E, R$ and $A$ prior to I-502 implementation (Jan 2011 - Sept 2012).

The set of data in Table 3 is then used to evaluate the right hand side function of system 1 .

\begin{tabular}{ccccc}
\hline \hline Variable & $\mathrm{N}$ & $\mathrm{E}$ & $\mathrm{R}$ & $\mathrm{A}$ \\
\hline Value & 42.947 & 46.643 & 7.558 & 2.851 \\
\hline
\end{tabular}

TABlE 3. Mean values for the state variables N, E, R and A prior to I-502 implementation (Jan 2011 - Sept 2012).

The fitness function over the period Jan 2011-Sept 2012 is computed as follows:

$$
\begin{aligned}
f\left(r_{1}, r_{2}, \cdots, r_{6}\right) & =\left(D_{1}-\left(\beta-\beta N-r_{1} N E-r_{1} N R+r_{3} E+r_{5} R+r_{6} A\right)\right)^{2} \\
& +\left(D_{2}-\left(r_{1} N E+r_{1} N R-r_{2} E R-\beta E-r_{3} E\right)\right)^{2} \\
& +\left(D_{3}-\left(r_{2} E R-\beta R-r_{4} R-r_{5} R\right)\right)^{2}+\left(D_{4}-\left(r_{4} R-\beta A-r_{6} A\right)\right)^{2},
\end{aligned}
$$

where $\beta=\frac{1}{15}$ and $D_{1}, D_{2}, D_{3}$ and $D_{4}$ represent the value of the temporal derivative for each category for the pre legalisation period. The resulting error given by Equation 4.5 is then minimised wrt the variables $r_{1}, \cdots, r_{6}$.

Incorporating the set of data from Tables 2 and 3 into Equation 4.5), the fitness function for the period prior to I-502 is obtained. Matlab Optimtool is employed and the fitness function, $f$ is inserted in the genetic algorithm, which minimises $f$ wrt $r_{1}, \cdots, r_{6}$. The following set of parameter values for the period prior the legalisation as given in Table 4, is thus yielded. 


\begin{tabular}{cccccccc}
\hline \hline Parameter & $r_{1}$ & $r_{2}$ & $r_{3}$ & $r_{4}$ & $r_{5}$ & $r_{6}$ & $\beta$ \\
\hline Value & $\mathbf{0 . 1}$ & $\mathbf{0 . 1 1 8}$ & 0.3 & 0.12 & 0.151 & 0.124 & $\frac{1}{15}$ \\
\hline
\end{tabular}

TABlE 4. Parameter values for the consumption of Marijuana in Washington prior to I-502 implementation (Jan 2011 - Sept 2012).

\begin{tabular}{ccccc}
\hline \hline Variable & $\mathrm{N}$ & $\mathrm{E}$ & $\mathrm{R}$ & $\mathrm{A}$ \\
\hline$D_{i}$ & -2.584 & 0.902 & 1.289 & 0.392 \\
\hline
\end{tabular}

TABle 5. Temporal derivatives for the state variables N, E, R and A after I-502 implementation (Nov 2012 - Dec 2015).

\begin{tabular}{ccccc}
\hline \hline Variable & $\mathrm{N}$ & $\mathrm{E}$ & $\mathrm{R}$ & $\mathrm{A}$ \\
\hline Value & 35.139 & 50.072 & 10.481 & 4.307 \\
\hline
\end{tabular}

TABle 6. Mean values for the state variables $\mathrm{N}, \mathrm{E}, \mathrm{R}$ and A after I-502 implementation (Nov 2012 - Dec 2015).

Similarly, the set of data from Tables 5 and 6 is used to evaluate Equation (4.5) in order to obtain the fitness function for the period after the I-502. The following set of parameter values for the period after the legalisation is obtained.

\begin{tabular}{cccccccc}
\hline \hline Parameter & $r_{1}$ & $r_{2}$ & $r_{3}$ & $r_{4}$ & $r_{5}$ & $r_{6}$ & $\beta$ \\
\hline Value & $\mathbf{0 . 2 9 7}$ & $\mathbf{0 . 1 2 2}$ & 0.1 & 0.12 & 0.111 & 0.132 & $\frac{1}{15}$ \\
\hline
\end{tabular}

TABLE 7. Parameter values for the consumption of Marijuana in Washington after I-502 implementation (Nov 2012 - Dec 2015).

From Tables 4 and 7, it is observed that the parameter values for $r_{1}$ and $r_{2}$ have both increased during the post legalisation period. It is noted that $r_{1}$ is the influence rate of $E(t)$ and $R(t)$ on $N(t)$ while $r_{2}$ represents the influence rate of $R(t)$ on $E(t)$. Despite the implementation of several public health campaigns funded from I-502, this observation indicates that $\mathrm{I}-502$ causes a rise in the influence that recreational users and experimental users exert on nonusers to try drugs in the post legalisation period. Legalisation inevitably encourages the tendency to try drugs that were otherwise illicit. This is a situation analogous to the increase of experimental users in Canada in the period just after the legalisation. Further, this study demonstrates that a 
campaign of prevention targeting both the experimental and recreational users is mandatory. As a result, this work gives insight on the type of campaign of prevention to be implemented, following legalisation in a certain population.

A second observation from Tables 4 and 7 is that the parameter value for $r_{4}$ remains the same for both periods. However, since the parameter values for $r_{1}$ and $r_{2}$ have increased in the post legalisation period, this account for a rise in the addict category as compared to the period prior to the I-502. Undoubtedly, this is confirmed by Figure 3 which depicts the rise in each of the three categories of drug users, post the legalisation.

Nevertheless, a drop in the parameter value for $r_{3}$ is accounted. In other words, less experimental users are quitting their category to join the nonuser class after the I-502 implementation. Consequently, this highlights the fact that campaigns of prevention focusing on the experimental and recreational categories is crucial in order to curb the transition of nonusers to the drug users' category.

Moreover, it is expected that after the legalisation of certain drugs, the nonusers will be tempted to try the drugs. Hence, this study highlights the importance of implementing targeted campaigns of prevention on the nonusers in order to thwart the value of $r_{1}$. The crucial sociological issue to address overhere is how to set up such a campaign of prevention. In such a campaign of prevention, the aim is to target the nonusers, the experimental users and the recreational users to take heed of the consequences of trying drugs.

For instance, campaigns of prevention with the aim of discouraging the first use of illicit drugs and disseminating the consequences of drug use are adequate. Recently, in September 2018, a new drug prevention campaign, 'Real Facts' has been set up with the objective of targeting youngsters aged 15 - 25 years old in Western Australia. The chief focus of the campaign are to raise awareness on the health effects of illicit drug use and prevent the use of such drugs [35]. In a certain sense, such a campaign of prevention is delaying the first use of drugs since they are directed to youngsters, in particular nonusers who are at their peak to try drugs. These results are provided with a view to support and give insights to decision makers in targeting prevention for maximum effectiveness in a given society.

Further, a campaign of prevention showing that there are much health awareness of relaxation than taking drugs. In [36], it is reported that sports represent an effective and optimal way to teach youngsters the healthy choices than illicit drug use. It is also stressed that there are individuals who practice sports to avoid the use of illicit drugs. Therefore, such a campaign of prevention that promotes recreational activities reduces 
the use of illicit drugs among the youth of the society. For example, the 'SPORT Prevention Plus Wellness' is a health promotion program for high school adolescents that lay emphasis on their physical activities, nutritional benefits and sleeping habits. Besides, this health program raises awareness on the health consequences of illicit drug use which diverge them from attaining their goals [37]. In fact, the enaction of such campaigns of prevention targeting the recreational and experimental users are crucial.

Figure 4 illustrates the different types of campaigns of prevention and the category that they effectively target. In an era of legalisation of drugs which were previously illicit, such targeted campaigns of prevention are important so as to effectively reduce $R_{0}$ and $\mu$ accordingly.

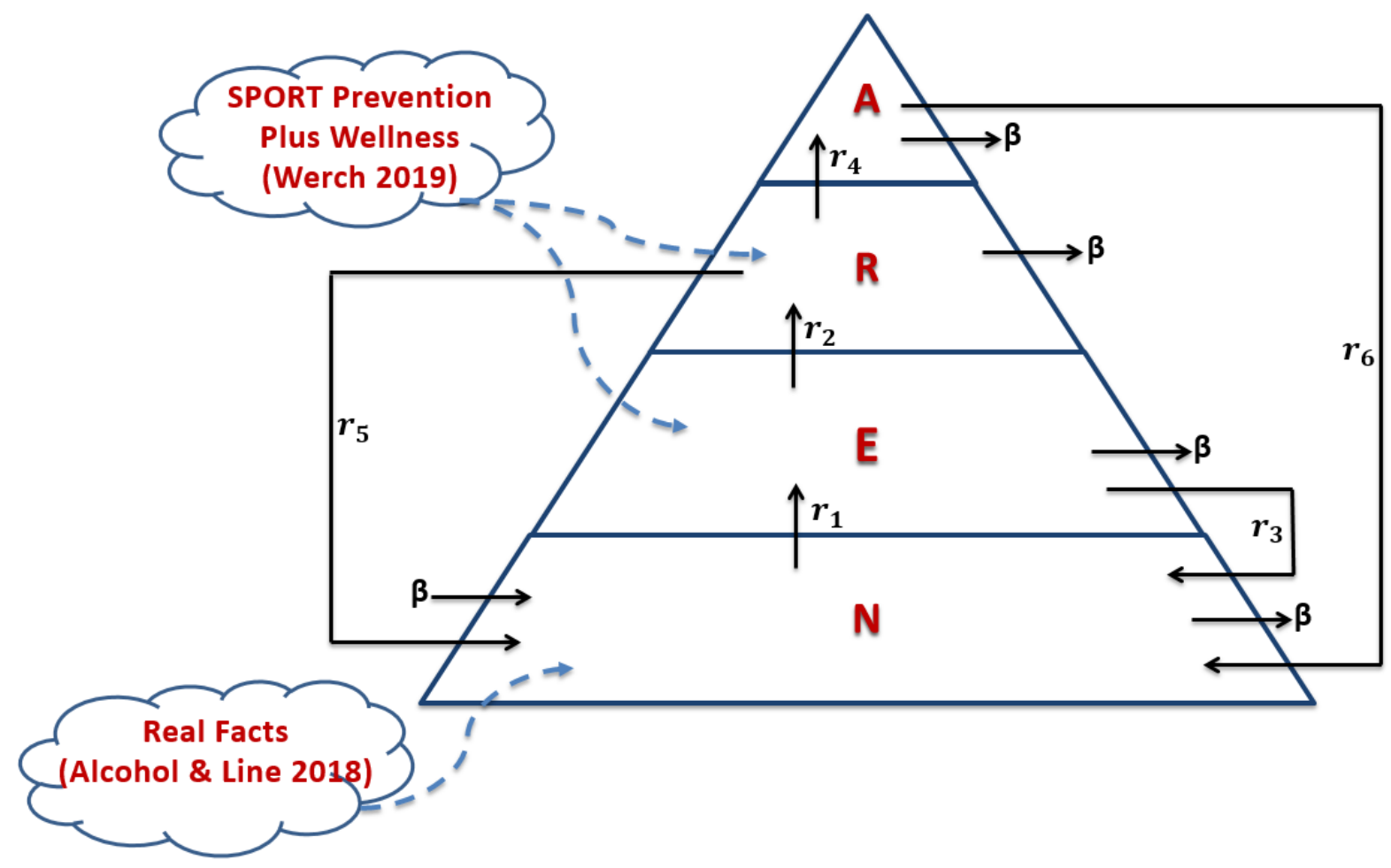

FigURE 4. The NERA model with campaigns of prevention targeting the nonusers, experimental users and recreational users respectively.

It is next proved that the set of parameter values given in Tables 4 and 7 which minimises function (4.1) and subjected to the constraints (4.3) and (4.4), gives the global minimum of function 4.1.

\subsection{Global minimum of the solution.}


Lemma 4.1. Let $f$ be a function of two or more variables with continuous first order and second order derivatives and the hessian of $f$ denoted by $H$, then $f$ is convex if and only if $H$ is positive semi definite.

Proposition 4.2. [38] Let $f: K \subset \mathbb{R}^{n} \rightarrow \mathbb{R}$ be continuous and $K$ be convex, we consider the problem $\min _{x \in K} f(x)$. If $f$ is convex, then every local minimum is a global minimum.

Proposition 4.3. 38] Let $f: \mathbb{R}^{n} \rightarrow \mathbb{R}$ be a continuous and coercive function and let $S \subseteq \mathbb{R}^{n}$ be a nonempty closed set. Then, $f$ has at least one global minimum point over $S$.

Theorem 4.4. The optimisation problem

$$
\underset{r_{j} \in \mathcal{F}}{\operatorname{minimise}} f\left(r_{1}, r_{2}, \cdots, r_{6}\right) \text {, }
$$

where $j=1,2, \cdots, 6$, has a global minimum with the following parameter set, $r_{j}=$ $\left\{r_{1}, r_{2}, \cdots, r_{6}\right\}$ on $\mathcal{F}$, where $f\left(r_{1}, r_{2}, \cdots, r_{6}\right)$ represents the objective function as defined in Equation (4.1) and given by

$$
f\left(r_{1}, r_{2}, \cdots, r_{6}\right)=\sum_{i=1}^{4}\left(D_{i}-P_{i}\right)^{2}
$$

with the constraint set

$$
\mathcal{F}=\left\{\left(r_{1}, r_{2}, \cdots, r_{6}\right) \in \mathbb{R}^{6} \mid \epsilon \leq \sum_{j=1}^{6} r_{j} \leq 1, \text { where } \epsilon>0 \text { and } 0<r_{j}<1\right\}
$$

for $j=1,2, \cdots, 6$.

Proof. It is observed that the objective function (4.7) is continuous and continuously differentiable. Firstly, it is shown that the objective function is convex. The Hessian of (4.7) is calculated and is given as

$$
\nabla^{2} f\left(r_{1}, r_{2}, \cdots, r_{6}\right)=
$$

$\left(\begin{array}{cccccc}4 N^{2}(R+E)^{2} & -2 E R N(R+E) & -4 E N(R+E) & 0 & -2 R N(R+E) & -2 A N(R+E) \\ -2 E R N(R+E) & 4 E^{2} R^{2} & 2 E^{2} R & -2 R^{2} E & -2 R^{2} E & 0 \\ -4 E N(R+E) & 2 E^{2} R & 4 E^{2} & 0 & 2 E R & 2 A E \\ 0 & -2 R^{2} E & 0 & 4 R^{2} & 2 R^{2} & -2 A R \\ -2 R N(R+E) & -2 R^{2} E & 2 E R & 2 R^{2} & 4 R^{2} & 2 A R \\ -2 A N(R+E) & 0 & 2 A E & -2 A R & 2 A R & 4 A^{2}\end{array}\right)$.


In reduced Row-Echelon form, the Hessian becomes

$$
\left(\begin{array}{cccccc}
4 N^{2}(R+E)^{2} & -2 E R N(R+E) & -4 E N(R+E) & 0 & -2 R N(R+E) & -2 A N(R+E) \\
0 & 3 E^{2} R^{2} & 0 & -2 R^{2} E & -3 R^{2} E & -E R A \\
0 & 0 & 0 & \frac{8}{3} R^{2} & 0 & -\frac{8}{3} A R \\
0 & 0 & 0 & 0 & 0 & 0 \\
0 & 0 & 0 & 0 & 0 & 0 \\
0 & 0 & 0 & 0 & 0 & 0
\end{array}\right),
$$

clearly the pivots are $\geq 0$. Hence, $\nabla^{2} f\left(r_{1}, r_{2}, \cdots, r_{6}\right)$ is positive semi definite and equivalently, using Lemma 4.1 , the function $f$ is convex.

It is next demonstrated that there exists a global minimum on $\mathcal{F}$.

From Section 3 , it is seen that the set $\Omega$ is closed and bounded, thus it is compact. Moreover, the constraint set $\mathcal{F}$ is also convex. Since $f$ is a convex function, by Proposition 4.2, every local minimum of $f$ is a global minimum on $\mathcal{F}$.

It is then sufficient to prove that the function, $f$, is coercive. Let $V=\left(r_{1}, r_{2}, \cdots, r_{6}\right)^{T}$ and set $t=\|V\|_{\infty}$.

The objective function is given as

$$
\begin{aligned}
f(t) & =\left(D_{1}-\beta+\beta N+t N R+t N E-t E-t R-t A\right)^{2} \\
& +\left(D_{2}-t N E-t N R+t E R+\beta E+t E\right)^{2} \\
& +\left(D_{3}-t E R+\beta R+2 t R\right)^{2}+\left(D_{4}-t R+\beta A+t A\right)^{2},
\end{aligned}
$$

and is bounded below as follows:

$$
f(t) \geq(-\beta-t E-t R-t A)^{2}+(-t N E-t N R)^{2}+(-t E R)^{2}+(-t R)^{2},
$$

where $\beta=\frac{1}{15}$, so that

$$
\lim _{t \rightarrow \infty} f(t)=\infty
$$

Therefore, $f$ is coercive. Further, since $f$ is in addition continuous, using Proposition 4.3. $f$ has at least one global minimum point. It is thus deduced that $f$ has a global minimum on $\mathcal{F}$.

In the section that tails, some numerical experiments are conducted to show the efficiency of the NERA model and demonstrate the effect of enacting campaigns of prevention, prior to I-502. 


\section{Numerical simulations}

In order to verify the model, data from Tables 4 and 7 are used to perform the numerical experiments. Figure 5 shows the results of the NERA model (solid lines) superimposed on the exact mean proportion of each category from [33] (dotted) for the period 2011-2015.

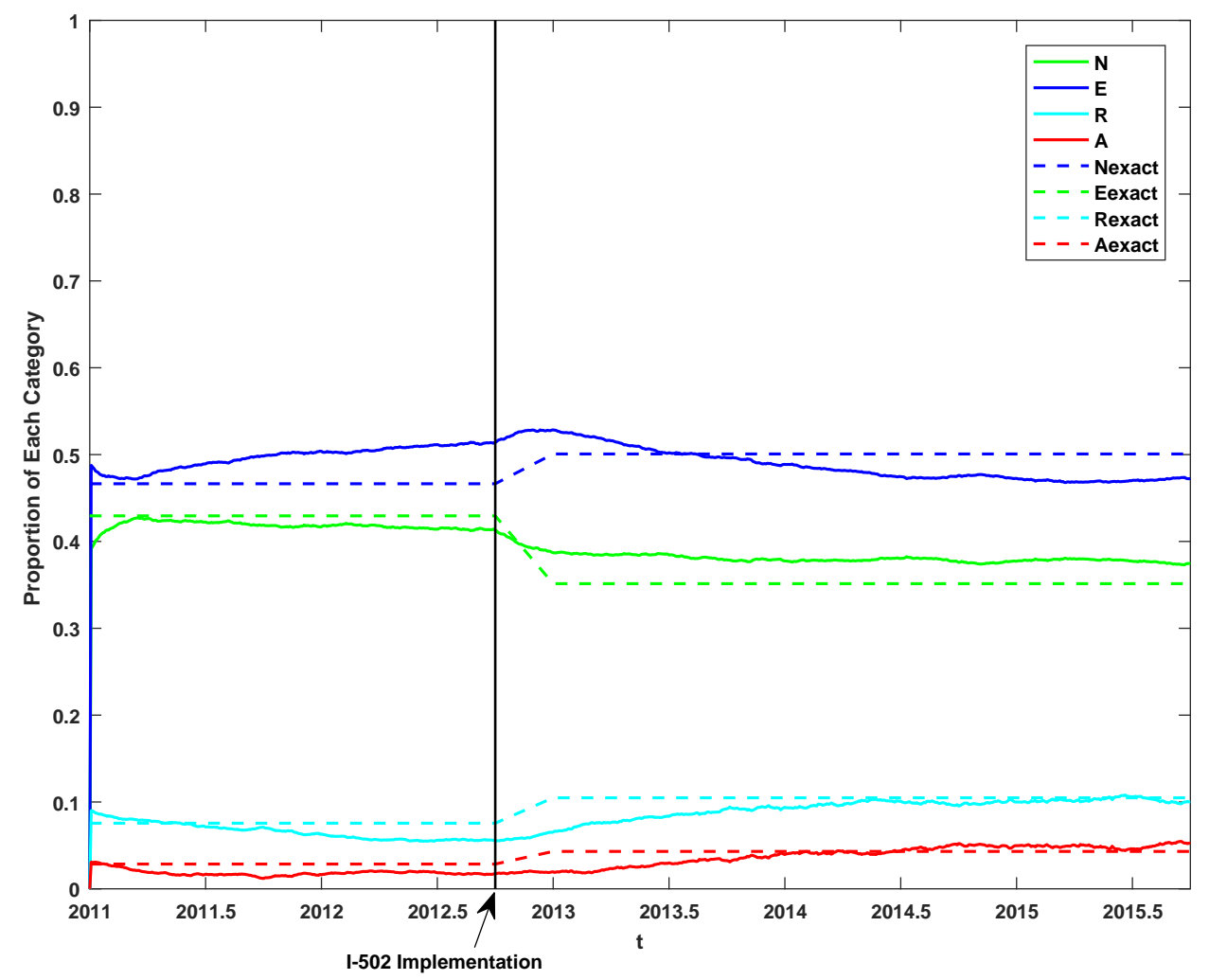

FIGURE 5. Superimposition of the evolution of N, E, R and A on the data collected from [33].

A comparison of the mean proportion of each category of users between the data gathered in [33] and the simulation of the model, for the periods before and after I-502 can be observed in Figures 6 and 7. Moreover, it should be pointed out that the NERA simulation agrees quite accurately with the data in [33]. This is illustrated in Table 8 which shows the $\%$ difference between the exact mean proportion of each category and that from the simulation of the NERA model, for the periods preceding and following I-502. 


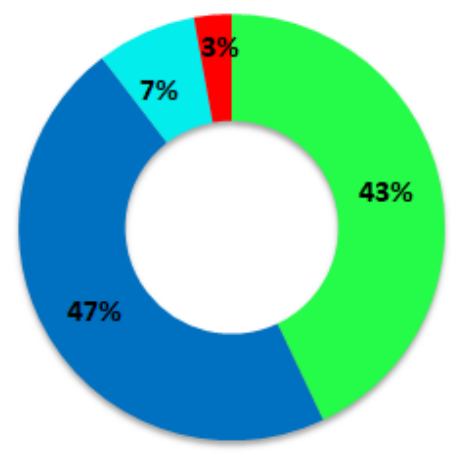

(a) Before I-502 (Jan 2011-Sept 2012).

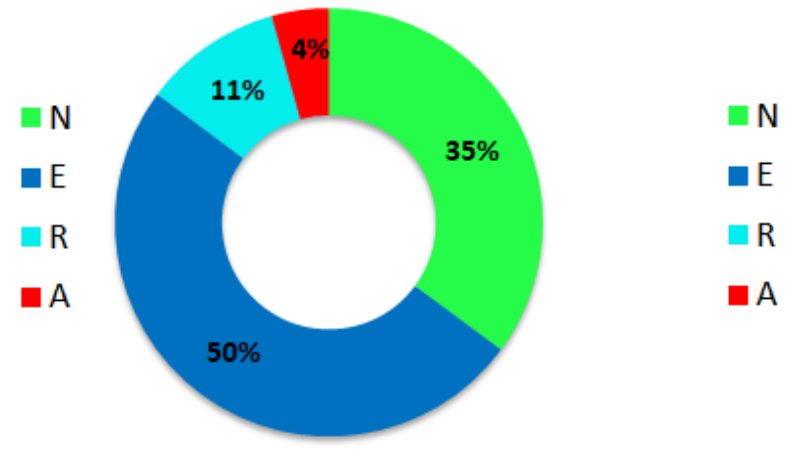

(b) After I-502 (Oct 2012-Dec 2015).

FiguRE 6. Mean proportion of each category for both periods according to data in [33].

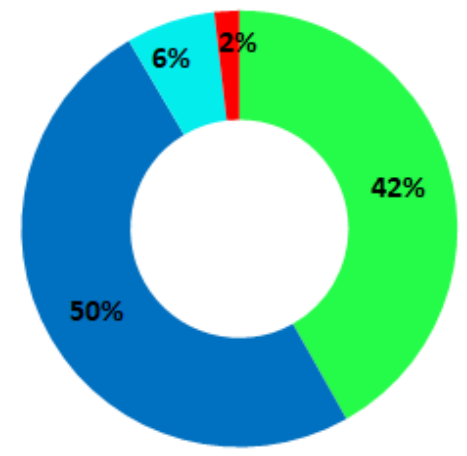

(a) Before I-502 (Jan 2011-Sept 2012).

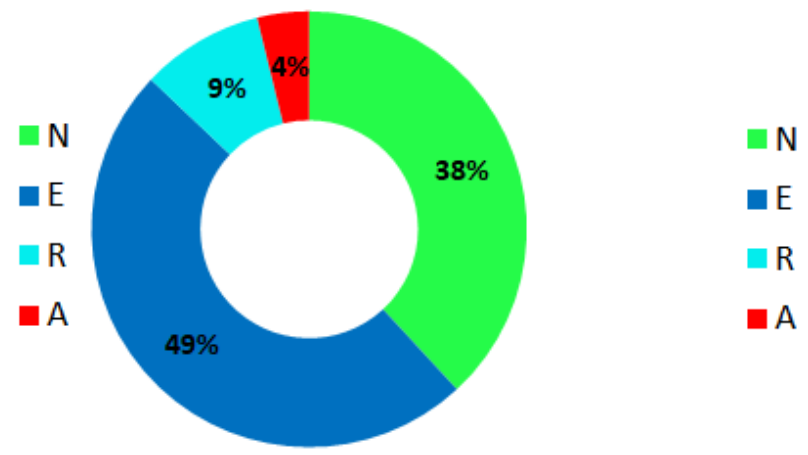

(b) After I-502 (Oct 2012-Dec 2015).

FiguRE 7. Mean proportion of each category for both periods according to the NERA model.

\begin{tabular}{ccccc}
\hline \hline Variable & $\mathrm{N}$ & $\mathrm{E}$ & $\mathrm{R}$ & $\mathrm{A}$ \\
\hline Pre & $1 \%$ & $3 \%$ & $1 \%$ & $1 \%$ \\
$(\mathrm{Jan} 2011$ - Sept 2012) & & & & \\
\hline Post & $3 \%$ & $1 \%$ & $2 \%$ & $0 \%$
\end{tabular}

(Oct 2012 - Dec 2015)

TABLE $8 . \%$ difference of the mean proportion of each category for both periods between the data in [33] and the simulation of the NERA model.

From Table 8, it is deduced that the simulation results of the NERA system show good agreement with the observed data confirming that the model can be a precious tool in forecasting illicit drug usage in a certain population. 
From the two sets of parameters as given in Tables 4 and 7 , the model reveals that during the post legalisation period, the parameter value $r_{1}$ and $r_{2}$ have increased. Consequently, this study gives a clear insight of the type of campaign of prevention to be implemented in a given population following legalisation. Hence, in the next section, the enactment of campaigns of prevention targeting experimental and recreational users, before and after I-502 is discussed.

\section{The effect of implementing campaigns of prevention 1 year before I-502}

One question that needs to be pointed out can be put as follows: what would have happened to the dynamics of marijuana consumption in Washington if campaigns of prevention were implemented prior to I-502?

Since the threshold number,

$$
R_{0}=\frac{r_{1}}{\beta+r_{3}},
$$

is defined as the average number of secondary experimental users generated by a typical drug user in the population, a decrease in $r_{1}$ leads to a decline in the number of individuals in the experimental class and an increase in the population of the nonuser class. However, an increase in $r_{3}$ causes a drop in the experimental category which in turn leads to a rise in the nonuser category.

In the NERA framework, an effective campaign of prevention amounts to one that targets both the experimental and recreational users in order to curb the initiation of marijuana usage. Thus, it is investigated how a reduction in both $r_{1}$ and $r_{2}$ and an increase in $r_{3}$ influence the dynamics of the model. The data given in Table 9 is used, it is the same information as in Table 4, except that $r_{1}$ and $r_{2}$ are decreased from 0.1 to 0.08 and 0.118 to 0.11 respectively and $r_{3}$ is increased from 0.3 to 0.33 .

\begin{tabular}{cccccccc}
\hline \hline Parameter & $r_{1}$ & $r_{2}$ & $r_{3}$ & $r_{4}$ & $r_{5}$ & $r_{6}$ & $\beta$ \\
\hline Value & $\mathbf{0 . 0 8}$ & $\mathbf{0 . 1 1}$ & $\mathbf{0 . 3 3}$ & 0.12 & 0.151 & 0.124 & $\frac{1}{15}$ \\
\hline
\end{tabular}

TABLE 9. Parameter values for a campaign of prevention on consumption of Marijuana in Washington.

Figure 8 pictures the evolution of the drug consumers when campaigns of prevention are implemented 1 year before the I-502 implementation. It is clear that the number of drug users is less than the mean exact trend (dotted lines). 


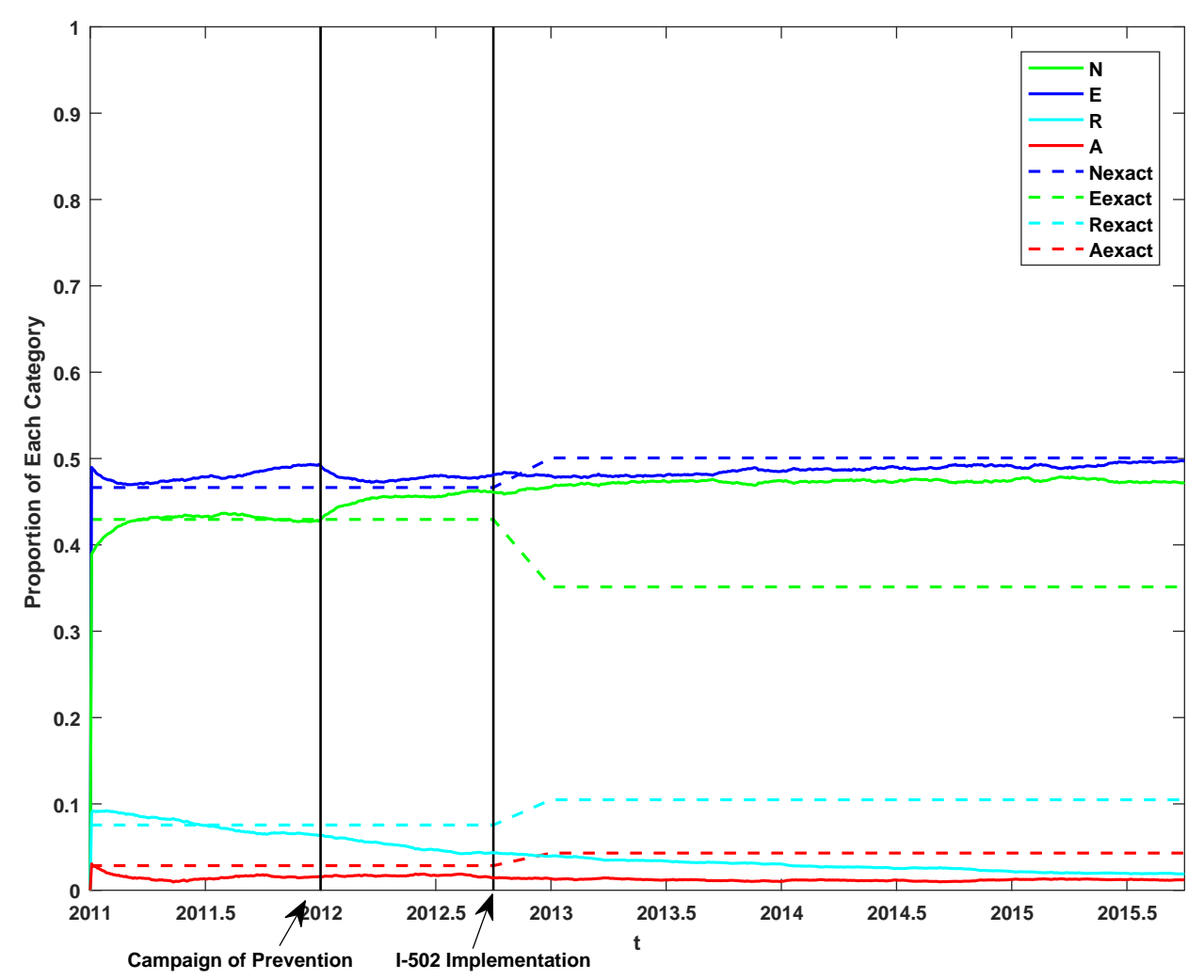

Figure 8. Evolution of N, E, R and A with campaigns of prevention initiated 1 year before I-502 Implementation.

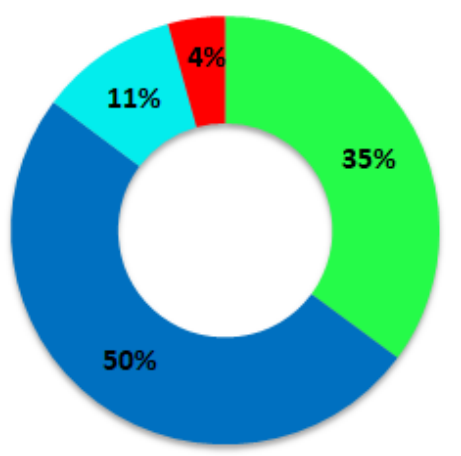

(a) Mean proportion of N, E, R and A according to data gathered in 33 .

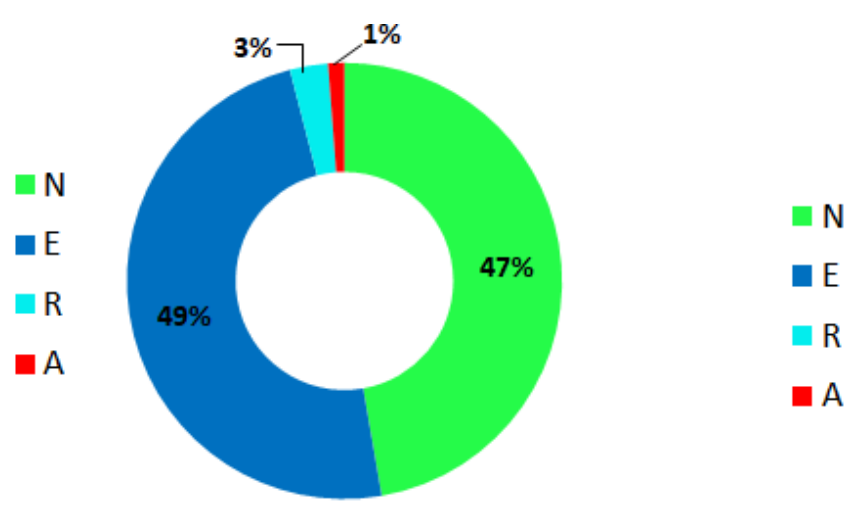

(b) Mean proportion of N, E, R and A when campaigns of prevention are initiated 1 year prior to I-502.

FiguRE 9. A comparison of the mean proportion of each category of users between the data gathered in [33] and the simulation of the NERA model, for the period post legalisation.

From Figure 9, it is seen that the number of nonusers is higher by $12 \%$ as compared to the proportion in Figure 9(a). A decrease of $1 \%$ among the experimental users is noted 
in the system. Moreover, it is illustrated that the population of recreational users has dropped drastically as compared to the proportion in Figure 9(a). A decrease of $8 \%$ is observed for the proportion of recreational users and a decrease of $3 \%$ among the addicts. The population of the addicts reduces due to the drop in the number of recreational individuals. This demonstrates the effectiveness of a campaign of prevention when targeting the experimental and recreational users 1 year before I502 implementation.

Since the recreational users are the advocates of drug consumption and they are those who stand the highest risk of becoming eventual addicts, it is crucial to limit this category from growing. Thus, suggested improvements include targeted campaigns of prevention, directed to both the experimental and recreational users in order to discourage the use of drugs. The NERA model supports this view firmly and shows the effect of implementing campaigns of prevention prior to I-502.

The system of differential equations given by system 3.1 is next used to forecast marijuana consumption in the State of Washington up to the year 2023 since the next report on the benefit-cost evaluation of I-502 will be produced in 2022 [33]. Figure 10 depicts the evolution of the three categories of marijuana users and the category of nonusers. It is seen that the proportion of experimental users is always higher than that of nonusers. Also, a remarkable increase is observed for the proportion of addicts. This simulation indicates that the legalisation of marijuana in the state of Washington encourages recreational consumption and may eventually leads to addiction.

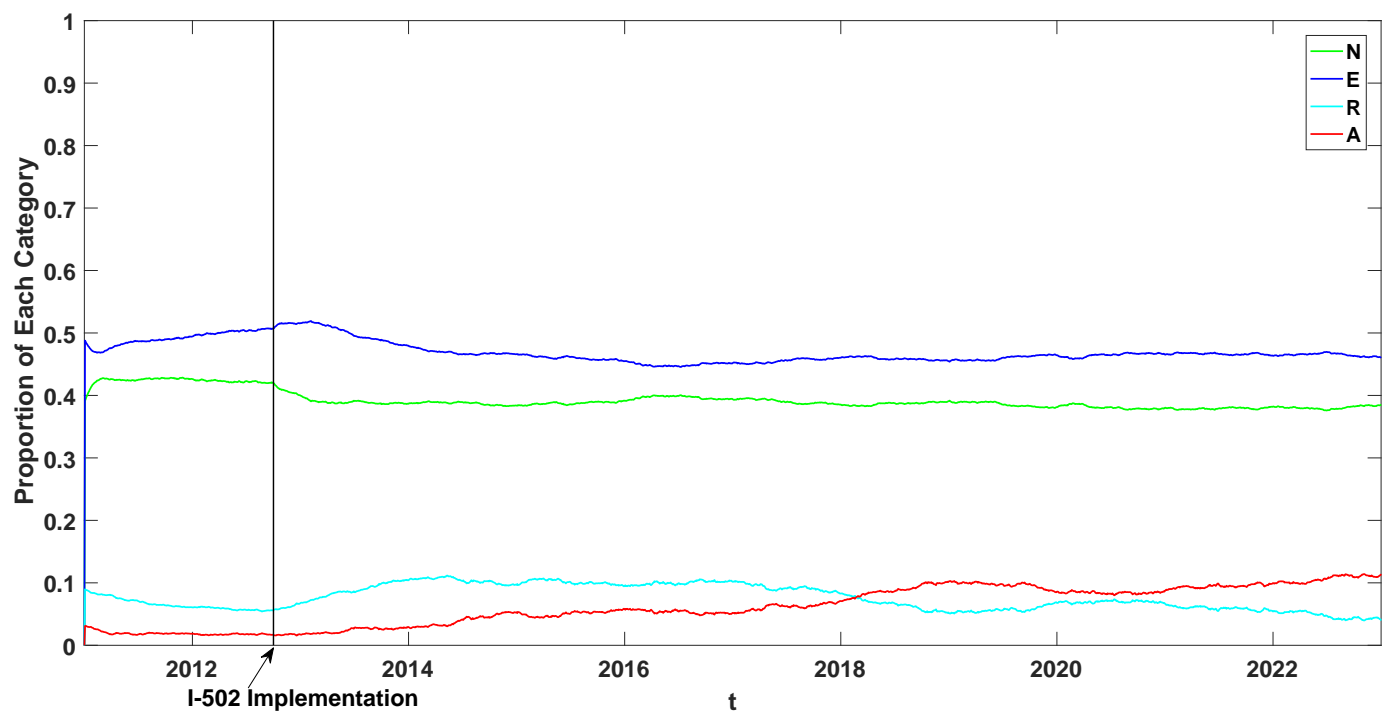

Figure 10. Evolution of N, E, R and A for the period 2011-2023. 


\section{Limitations of the NERA model and the present work}

The movement out of the population due to natural death is not taken into account in the NERA model. A number of researchers have investigated on the lethal rates of marijuana use and found that it has the lowest risk of mortality [39]. Besides, it is assumed that the influence rate of the experimental and recreational users on the nonusers are equal to $r_{1}$. This can be addressed in a future study by having two different influence rates on the nonuser category.

We point out that in the present work, only one targeted campaign of prevention is conducted. The latter scenario analysis highlights the insight that NERA can give in deciding about the type of campaign of prevention and the corresponding impact on the proportion of marijuana users. In a future work, more 'what if' scenario campaigns of prevention on the nonuser category and different other possible combinations of categories such as nonuser and recreational users can be effectuated. Further, the NERA system has been verified over the period 2011 to end of 2015. With the availability of new data for the state of Washington, till 2023, the model can be validated in a future work.

In the present work, it has been forecasted that by 2023 there will be more experimental users than nonusers among the population of $21+$ and that the number of addicts may exceed the population of recreational users. Although it is difficult to quantify exact proportion of nonusers, experimental and regular marijuana users, still the model is very useful because it gives an indication of the trend of marijuana consumption by 2023.

\section{Conclusion}

In this work, the NERA model was used to assess the effect of I-502 implementation in the state of Washington. The permanence of the system was shown when $R_{0}>1$ and $\mu>1$. Data gathered in [33] was considered for the verification of the model using genetic algorithm for the periods pre and post I-502. The simulation results had shown good agreement with the available data. The numerical results indicated a rise in the influence that recreational users and experimental users exert on nonusers to try drugs in the post legalisation period. The present work is a clear evidence that legalisation enhances the recreational use and eventually lead to an abuse of marijuana amongst the $21+$ population of the state of Washington. The numerical experiments demonstrated clearly that the aftermath of legalisation without proper campaigns of prevention, is an abusive consumption of marijuana. The NERA model pointed out that targeted campaigns of prevention enacted 1 year before I-502, would have decreased the experimental, recreational and addict categories by $1 \%, 8 \%$ and $3 \%$ respectively. This study sheds light on the type of campaign of prevention to be 
implemented in order to help policy and decision makers to curtail abusive marijuana use.

\section{Acknowledgments}

The authors would like to acknowledge the assistance of Mr Maheshsingh Mungur from the University of Mauritius in the preparation of this work. This work has been conducted under the HEC (Higher Education Commission Mauritius) MPhil/PhD scholarship.

\section{REFERENCES}

[1] A. Dugar, Differences Between Medical Cannabis vs Recreational Marijuana, date accessed: 26 12 19, https://greenhealthdocs.com/medical-vs-recreational-marijuana/ (2019).

[2] Y.-W. L. Chu, The effects of medical marijuana laws on illegal marijuana use, Journal of Health Economics 38 (2014) 43-61.

[3] ProCon.org, Peer-reviewed studies on medical marijuana, date accessed: $27 \quad 12$ 19, https://medicalmarijuana.procon.org/peer-reviewed-studies-on-medical-marijuana/ (2016).

[4] B. Mastroianni, Why do most patients use medical marijuana? chronic pain, date accessed: 2712 19, https://www.healthline.com/health-news/what-drives-patients-to-usemedical-marijuana-chronic-pain (2019).

[5] N. L. Health, NYU School of Medicine, In states where recreational marijuana is legal, problematic use increased among adults and teens., date accessed: $27 \quad 12 \quad 19$, www.sciencedaily.com/releases/2019/11/191113153049.htm (2019).

[6] G. Canada, A framework for the legalization and regulation of cannabis in canada, the final report of the task force on cannabis legalization and regulationDate accessed: 20 October 2018. URL https://www.canada.ca/en/health-canada/services/drugs-medication/ cannabis/laws-regulations/task-force-cannabis-legalization-regulation/ framework-legalization-regulation-cannabis-in-canada.html

[7] A. Brooks-Russell, M. Ma, A. H. Levinson, L. Kattari, T. Kirchner, E. M. A. Goodell, R. M. Johnson, Adolescent marijuana use, marijuana-related perceptions, and use of other substances before and after initiation of retail marijuana sales in colorado (2013-2015), Prevention science (2018) 1-9.

[8] S. Hanley, Legalization of recreational marijuana in Washington: Monitoring trends in use prior to the implementation of I-502.Report ID: 13-11-1401. [online] Washington: Washington State Institute for Public Policy. Available at: http://www.wsipp.wa.gov/Reports/526 [Accessed 16 Apr. 2018].

[9] Y. M. Prevention, Youth marijuana prevention, gmmbDate accessed: 23 November 2018. URL https://gmmb.com/work/youth-marijuana-prevention

[10] Listen2YourSelfie, Washington kicks-off youth marijuana prevention campaignDate accessed: 23 November 2018.

URL https://www.doh.wa.gov/Newsroom/2016NewsReleases/16042MarijuanaCampaign

[11] K. C. S. Health, Doh launches "you can" marijuana prevention and education campaignDate accessed: 23 November 2018.

URL https://kingcountyschoolhealth.com/2018/04/09/doh-launches-you-can-marijuana-prevention-and 
[12] P. D. F. Kids, The partnership attitude tracking studyDate accessed: 23 November 2018. URL https://drugfree.org/wp-content/uploads/2014/07/PATS-2013-FULL-REPORT.pdf

[13] R. J. Haines-Saah, S. Mitchell, A. Slemon, E. K. Jenkins, 'parents are the best prevention'? troubling assumptions in cannabis policy and prevention discourses in the context of legalization in canada, International Journal of Drug Policy.

[14] T. Wiens, K. M. Lenk, L. E. Fabian, D. J. Erickson, Law enforcement practices in the first two states in us to legalize recreational marijuana, International Journal of Drug Policy 61 (2018) $38-43$.

[15] E. Hyshka, Applying a social determinants of health perspective to early adolescent cannabis use-an overview, Drugs: education, prevention and policy 20 (2) (2013) 110-119.

[16] V. Channels, Canada is already experiencing cannabis shortagesDate accessed: 20 October 2018. URL https://www .vice.com/en-ca/article/evw99z/canada-is-already-experiencing-cannabis-shortages

[17] J. Canada, Cannabis legalization and regulation - justice canadaDate accessed: 20 October 2018 .

URL http://www.justice.gc.ca/eng/cj-jp/cannabis/

[18] Y. B. Ruhomally, N. B. Jahmeerbaccus, M. Z. Dauhoo, The deterministic evolution of illicit drug consumption within a given population, ESAIM: Proceedings and Surveys 62 (2018) 139157.

[19] M. Santoro, L. Triolo, C. Rossi, Drug user dynamics: A compartmental model of drug users for scenario analyses, Drugs: education, prevention and policy 20 (3) (2013) 184-194.

[20] C. Rossi, Operational models for epidemics of problematic drug use: the mover-stayer approach to heterogeneity, Socio-Economic Planning Sciences 38 (1) (2004) 73-90.

[21] H. W. Hethcote, The basic epidemiology models: models, expressions for r0, parameter estimation, and applications, in: Mathematical understanding of infectious disease dynamics, World Scientific, 2009, pp. 1-61.

[22] M. Dauhoo, B. Korimboccus, S. Issack, On the dynamics of illicit drug consumption in a given population, IMA J Appl Math 78 (3) (2013) 432-448.

[23] N.-R. Badurally Adam, M. Z. Dauhoo, O. Kavian, An analysis of the dynamical evolution of experimental, recreative and abusive marijuana consumption in the states of colorado and washington beyond the implementation of i-502, The Journal of Mathematical Sociology 39 (4) (2015) 257-279.

[24] Y. B. Ruhomally, M. Z. Dauhoo, The NERA model incorporating cellular automata approach and the analysis of the resulting induced stochastic mean field, Computational and Applied Mathematics 39 (4) (2020) 1-29.

[25] W. O. Kermack, A. G. McKendrick, A contribution to the mathematical theory of epidemics, Proceedings of the royal society of london. Series A, Containing papers of a mathematical and physical character 115 (772) (1927) 700-721.

[26] N. Sherborne, K. B. Blyuss, I. Z. Kiss, Dynamics of multi-stage infections on networks, Bulletin of mathematical biology 77 (10) (2015) 1909-1933.

[27] M. M. of Pathogenicity, Characteristics of infectious disease, date accessed: 19 06 20, https://courses.lumenlearning.com/microbiology/chapter/characteristics-of-infectiousdisease/ (2016).

[28] V. Wang, Most coronavirus cases are mild. that's good and bad news., https://www.nytimes.com/2020/02/27/world/asia/coronavirus-treament-recovery.html (2020).

[29] I. Deibe, Coronavirus recovery: How do people recover?, https://www.express.co.uk/lifestyle/health/1258191/coronavirus-recovery-how-do-people-recover-covid-19 (2020). 
[30] D. Korf, Trends and patterns in cannabis use in the Netherlands, Paper to be presented at the Hearing of the Special committee on Illegal Drugs. OttawaHttps://sencanada.ca/content/sen/committee/371/ille/presentation/korf-e.htm.

[31] L. Wang, Z. Teng, H. Jiang, Global attractivity of a discrete sirs epidemic model with standard incidence rate, Mathematical Methods in the Applied Sciences 36 (5) (2013) 601-619.

[32] X.-Q. Zhao, Dynamical systems in population biology, Springer Science \& Business Media, 2013.

[33] A. Darnell, K. Bitney, I - 502 evaluation and benefit-cost analysis: Second required report, washington state institute for public policy. (2017), Technical Report.

[34] B. Song, M. Castillo-Garsow, K. R. R ios Soto, M. Mejran, L. Henso, C. Castillo-Chávez, Raves, clubs and ecstasy: the impact of peer pressure, Mathematical Biosciences and Engineering 3 (1) (2006) 249.

[35] Alcohol, D. S. Line, Drug aware, real facts, date accessed: $\begin{array}{llll}12 & 09 & 19 \text {, }\end{array}$ https://drugaware.com.au/about-us/current-campaigns/real-facts-about-drugs/ (2018).

[36] A. McKiernan, Youth sport programs that address substance use - an environmental scan, canadian centre on substance abuse, date accessed: 1209 19, https://www.ccsa.ca/youth-sportprograms-address-substance-use-environmental-scan (2016).

[37] C. Werch, Sport prevention plus wellness, date accessed: $\begin{array}{llll}12 & 09 & 19 \text {, }\end{array}$ https://www.blueprintsprograms.org/programs/sport-prevention-plus-wellness/ (2019).

[38] A. Beck, Introduction to nonlinear optimization: theory, algorithms, and applications with MATLAB, Vol. 19, Siam, 2014.

[39] M. Ferner, Marijuana may be the least dangerous recreational drug, study showsHttps://www.huffingtonpost.com/2015/02/24/marijuana-safer-than-alcohol-tobaccon6738572.html. 